\title{
Depressive symptoms in elderly women: knowing them to care for*
}

\author{
Maria do Livramento Fortes Figueiredo ${ }^{1}$
}

The increased life expectancy associated with the improved health conditions has resulted in the increasing and ongoing rise of the elderly population in most societies. Projections show that, by the year 2050, there will be approximately two billion older people across the world, most of who will be living in developing countries. Population aging in Brazil is a phenomenon derived from technological advances in the field of health, particularly the reduced child mortality, the decreased fertility due to the introduction of contraceptive methods, the expanded vaccination coverage and the use of antibiotics and chemotherapy drugs that enabled the prevention and healing of many diseases. Although old age is a universal process, its experience has a great distinction by gender, mainly because there is higher number of elderly women, as aging increases. This phenomenon is characterized as the feminization of old age, adding to this the fact that there is the onset of several chronic diseases, among them depression and its symptomatology.

The depressive syndrome is characterized by the presence of a predominantly depressive and/or grumpy mood and anhedonia (decreased ability to feel pleasure or joy). Furthermore, there may still be a subjective sensation of decreased energy (tiredness and fatigue), disinterest, slowing down, pessimistic thoughts and ideas of depletion. In general, these symptoms can be followed by changes in sleep and appetite, cognitive impairment, behavioral changes and physical symptoms.

Epidemiologically, older women have relevant characteristics that make them more vulnerable in old age, among them: higher propensity for diseases and problems related to adaptation to physiological changes due to age, which are worsened by poverty, multiple pregnancies, physical and psychological wear arising from tough working days, lack of physical activity, in addition to their social and economic subservience to the male figure.

The findings of this study also unveiled the association of depressive symptoms with the family organization experienced by the research participants and the longer time of retirement, as well as the perception of the elderly women when they compared their economic condition with the condition of other people of the same age. The qualitative stage of the survey took place through household interviews with the elderly women who showed more significant depressive symptomatology, which unveiled that the leading causes of these symptoms were: feeling of neglect, loneliness and contempt; losses of children; problems with offspring and also the presence of diseases.

Given this evidence, it is highlighted the importance of the creation and implementation of programs for including elderly women in sociocultural and leisure movements; as well as the deployment of the follow-up of depressive symptoms in the daily routine of Primary Care; and also the qualification of health professionals, mainly nurses, in order to develop a health care based on the comprehensive knowledge of the human aging process. Accordingly, it is proposed to assist these customers by meeting their needs and demands, with a view to providing health promotion, disease prevention, early diagnosis, suitable treatment and effective rehabilitation. Lastly, it is believed that the knowledge of depressive symptoms in Primary Care will enable the comprehensive and multidimensional care of the elderly women assisted by the Family Health Strategy.

\footnotetext{
*Extracted from the doctoral thesis in nursing "Prevalência de sintomas depressivos e fatores associados em mulheres idosas assistidas na Atenção Básica, na perspectiva do Curso de Vida", authorship of Jaqueline Carvalho e Silva Sales, 2016. Available from: http://repositorio. ufpi.br/xmlui/bitstream/handle/123456789/459/TESE\%20\%20Jaqueline_Carvalho.pdf?sequence=1

${ }^{1}$ Universidade Federal do Piauí. Teresina, PI, Brazil. 